\title{
Inhibition of microRNA-29b reduces murine abdominal aortic aneurysm development
}

\author{
Lars Maegdefessel, ${ }^{1}$ Junya Azuma, ${ }^{1}$ Ryuji Toh, ${ }^{1}$ Denis R. Merk, ${ }^{2}$ Alicia Deng, ${ }^{1}$ Jocelyn T. Chin, ${ }^{2}$ \\ Uwe Raaz, ${ }^{1}$ Anke M. Schoelmerich, ${ }^{1}$ Azad Raiesdana, ${ }^{1}$ Nicholas J. Leeper, ${ }^{1}$ Michael V. McConnell, ${ }^{1}$ \\ Ronald L. Dalman, ${ }^{3}$ Joshua M. Spin, ${ }^{1}$ and Philip S. Tsao ${ }^{1}$
}

1Division of Cardiovascular Medicine, ${ }^{2}$ Department of Cardiothoracic Surgery, and ${ }^{3}$ Division of Vascular Surgery, Stanford University School of Medicine, Stanford, California, USA.

\begin{abstract}
MicroRNAs (miRs) regulate gene expression at the posttranscriptional level and play crucial roles in vascular integrity. As such, they may have a role in modifying abdominal aortic aneurysm (AAA) expansion, the pathophysiological mechanisms of which remain incompletely explored. Here, we investigate the role of miRs in 2 murine models of experimental AAA: the porcine pancreatic elastase (PPE) infusion model in C57BL/6 mice and the AngII infusion model in $\mathrm{Apoe}^{-/-}$mice. AAA development was accompanied by decreased aortic expression of miR-29b, along with increased expression of known miR-29b targets, Col1a1, Col3a1, Col5a1, and Eln, in both models. In vivo administration of locked nucleic acid anti-miR-29b greatly increased collagen expression, leading to an early fibrotic response in the abdominal aortic wall and resulting in a significant reduction in AAA progression over time in both models. In contrast, overexpression of miR-29b using a lentiviral vector led to augmented AAA expansion and significant increase of aortic rupture rate. Cell culture studies identified aortic fibroblasts as the likely vascular cell type mediating the profibrotic effects of miR-29b modulation. A similar pattern of reduced miR-29b expression and increased target gene expression was observed in human AAA tissue samples compared with that in organ donor controls. These data suggest that therapeutic manipulation of miR$29 \mathrm{~b}$ and its target genes holds promise for limiting AAA disease progression and protecting from rupture.
\end{abstract}

\section{Introduction}

Abdominal aortic aneurysms (AAAs) occur in approximately $9 \%$ of patients older than 65 years of age (1). The most feared clinical consequence of AAA progression is acute rupture, which accounts for about 15,000 deaths annually in the United States (2). Risk factors for AAA development include cigarette smoking, hypertension, male sex, and a family history of AAA in a male first-degree relative as well as presence of atherosclerosis in other vascular beds (3). Epidemiologic and pathologic studies have provided few clues to the causes of AAA development, progression, and ultimate rupture. Pathologic features of aneurysm include smooth muscle cell apoptosis, local inflammation, increased oxidative stress, and significant matrix degradation (4).

MicroRNAs (miRs) are approximately 20 -nucleotide, singlestranded RNA molecules that target mRNA through partial complementarity and regulate gene expression through inhibition of translation or transcript degradation (5). Their roles in AAA disease as well as their therapeutic potential to inhibit aneurysm expansion have been minimally explored to date.

The miR-29 family (miR-29a, miR-29b, and miR-29c) has been identified previously to promote fibrosis through regulation of its downstream target genes (6-8). Validated targets include a large number of collagen isoforms, many of which are expressed in the abdominal aorta and have been linked to aneurysm formation (9-11). These include collagen, type I, $\alpha 1$ (COL1A1), COL3A1, and COL5A1 as well as other key components of the aortic wall, such as fibrillin-1 (FBN1) and elastin (ELN). MMP2 and MMP9, both associated with AAA development and progression in previous studies (12), have been identified as direct targets of miR-29b (13).

Conflict of interest: Lars Maegdefessel, Joshua M. Spin, and Philip S. Tsao have applied for a patent relating to this work.

Citation for this article: J Clin Invest. 2012;122(2):497-506. doi:10.1172/JCI61598.
We hypothesized that altering expression of the miR-29 family and its putative gene targets would affect extracellular matrix remodeling, allowing us to modify AAA disease progression and thereby potentially offer a novel molecular therapy.

\section{Results}

miR-29b is significantly downregulated and negatively correlated with its target genes during aneurysm development in 2 murine models of $A A A$. Using the porcine pancreatic elastase (PPE) infusion model in 10week-old male C57BL/6J mice (14), we investigated the expression of the miR-29 family and its putative target genes at multiple time points during infrarenal AAA development.

B-mode ultrasound imaging performed 3, 7, 14, 21, and 28 days after PPE infusion demonstrated the expected progressive increases in abdominal aortic diameter (AAD) in elastase-infused (ELAST) mice compared with those in saline-infused control (sham) mice (Figure 1A and Supplemental Table 1; supplemental material available online with this article; doi:10.1172/JCI61598DS1). Ex vivo diameters at necropsy (after 7, 14, and 28 days) were analyzed through direct measurement; the diameters closely correlated with AADs obtained with ultrasound (data not shown).

Infrarenal aortic tissue was harvested at days 7, 14, and 28 . Interestingly, miR-29b was the only miR-29 family member that was significantly downregulated at all 3 time points. miR-29c and miR-29a trended downward at all time points, but the only significant difference compared with sham-operated mice was miR-29c at 7 days (Figure 1B).

The miR-29 family is transcribed as 2 bicistronic primary miRs, located on 2 different murine chromosomes: primary-miR (primiR) 29b-1/29a is located on chromosome 6, and pri-miR-29b$2 / 29 \mathrm{c}$ is located on chromosome 1 . Both pri-miRs were significantly downregulated (between -1.55 to -1.84 fold; $P<0.05$ ) at 

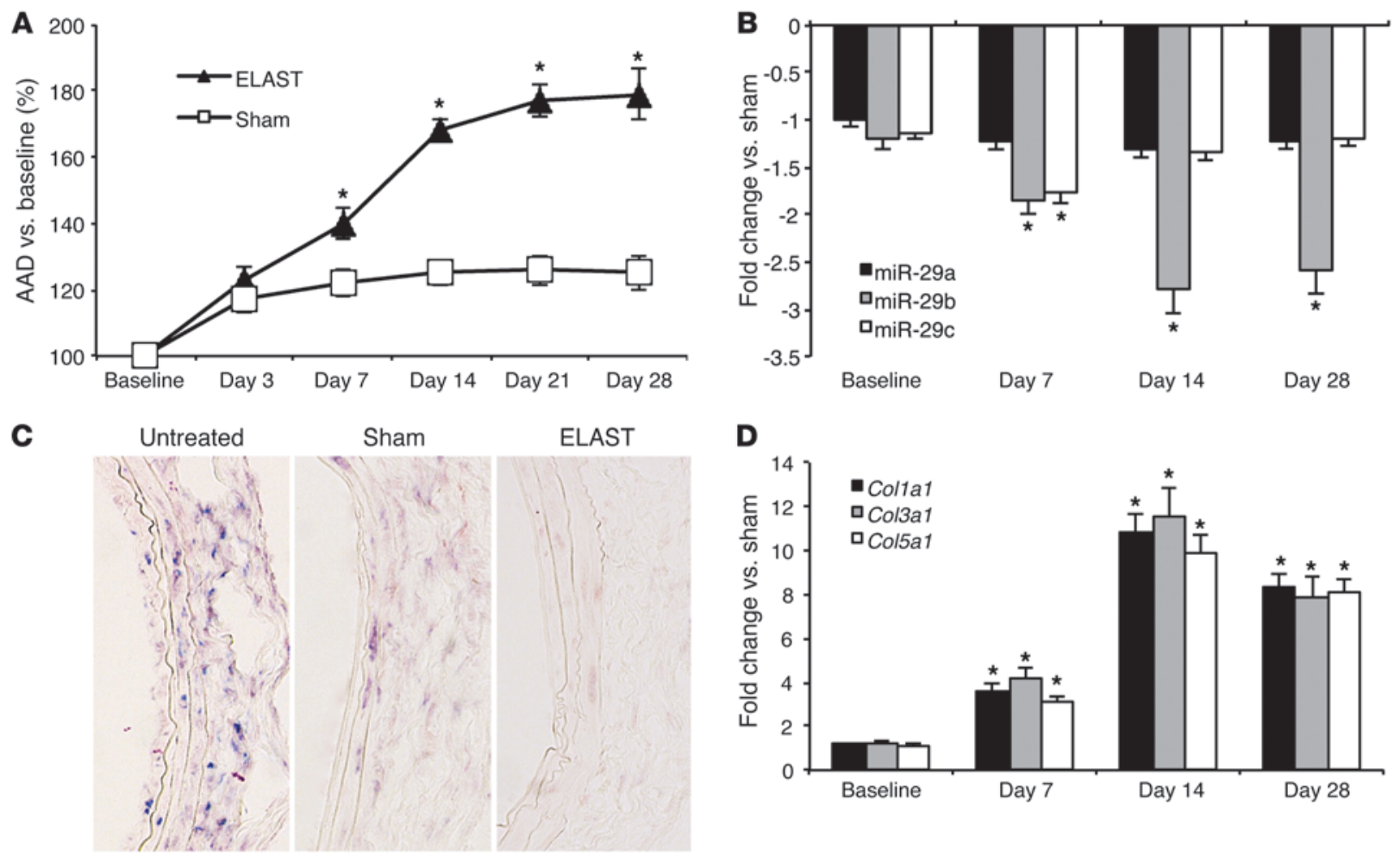

D
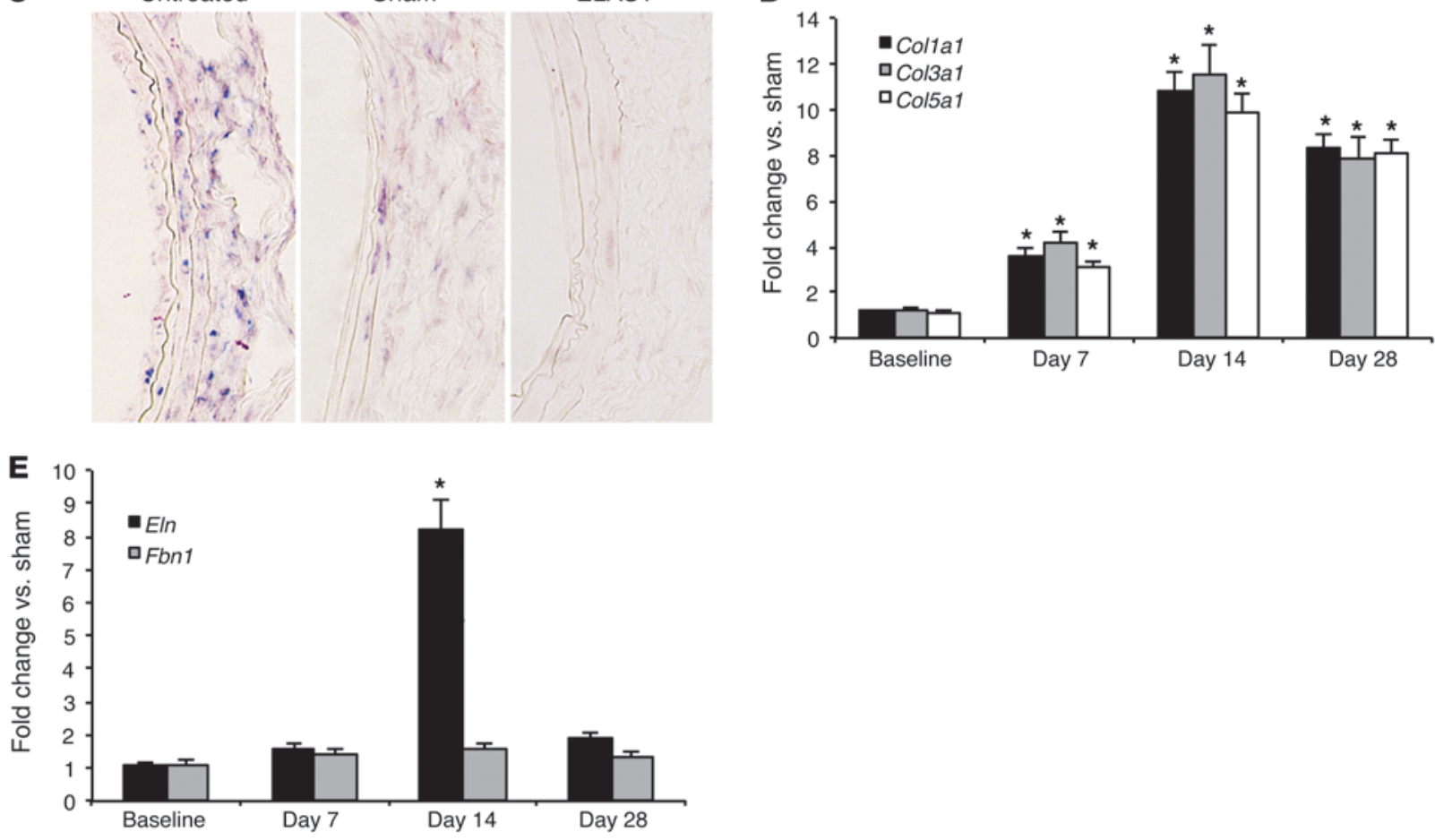

Figure 1

miR-29 in AAAs induced by PPE infusion in mice. (A) AAD (versus baseline in percentage) in PPE-induced (ELAST) AAA compared with that in sham-operated control mice (sham). (B) miR-29 family (miR-29a, miR-29b, miR-29c) expression in ELAST mice compared with that in sham-operated control mice. (C) ISH for miR-29b (purple chromogen) in control aortas (untreated), sham-operated mice, and ELAST mice 14 days after surgery (original magnification, $\times 200$ ). (D and E) mRNA expression levels in ELAST mice compared with those in sham-operated mice for (D) Col1a1, Col3a1, and Col5a1 as well as (E) Eln and Fbn1. $n=5-8$ for each treatment group and time point. Data are mean \pm SEM. ${ }^{\star} P<0.05$ versus sham.

all 3 time points (days 7, 14, and 28) during AAA expansion in the PPE model compared with those in sham-operated mice. These combined results suggest some level of posttranscriptional degradation of miR-29a and miR-29c when compared with miR-29b.

In situ hybridization (ISH) indicated that miR-29b expression was diminished throughout the aneurysmal aortic wall of ELAST mice compared with that of sham and untreated controls (Figure $1 \mathrm{C})$, particularly in the adventitial layer. Collagen gene expression (Col1a1, Col3a1, Col5a1) was negatively correlated with miR$29 \mathrm{~b}$ expression, and both effects peaked at day 14 (Figure 1D). Eln was significantly upregulated at 14 days after infusion but not at the other 2 time points. Notably, Fbn1 was not differentially regulated at any of the 3 time points, suggesting additional transcriptional modulators (Figure 1E).
We used a second established model of AAA formation (this one was suprarenal) with AngII infusion in 10-week-old Apoe-/- male mice (15) to confirm that aneurysm-related regulation of miR-29b and its ECM-related target genes was not exclusive to the PPE model. Compared with that in sham-operated mice, the AAD was significantly increased in the AngII group from day 7 through day 28 (Figure 2A and Supplemental Table 2). As expected, the mortality rate through day 28 was significantly $(P<0.01)$ higher in AngII mice $(26 \%)$, due to aortic rupture and dissection, when compared with that of saline controls $(0 \%)$. miR-29b was the only member of the miR-29 family that was significantly downregulated at 2 different time points during aneurysm development (days 14 and 28) in AngII mice compared with saline-infused control mice (Figure $2 \mathrm{~B}$ ). In this model, miR-29b expression was negatively correlated with expression of 

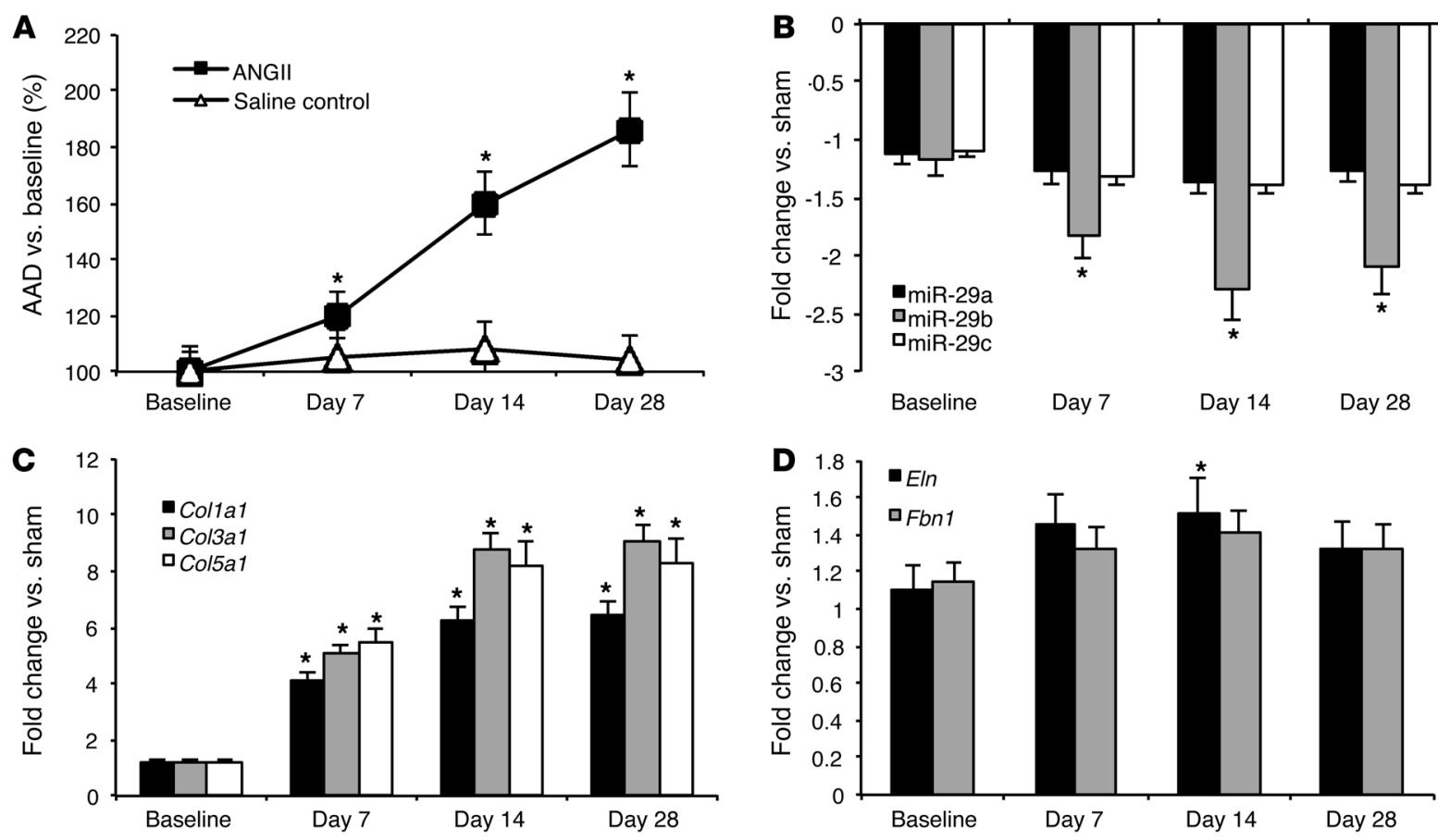

Figure 2

miR-29 and target gene expression in Angll-induced AAAs. (A) Expansion of AADs (in percentage of baseline) in mice with Angll-induced AAA (ANGII; $n=27$ ) compared with that in saline-infused controls (sham; $n=12$ ). (B) miR-29 expression in mice with Angll-induced AAA compared with that in sham-operated mice. (C) Col1a1, Col3a1, and Col5a1 expression in mice with Angll-induced AAA compared with that in sham-operated mice. (D) Eln and Fbn1 expression in mice with Angll-induced AAA compared with that in sham-operated mice. Data are mean \pm SEM. ${ }^{*} P<0.05$ versus untreated.

Col1a1, Col3a1, Col5a1, Eln, and Fbn1 (Figure 2, C and D). However, only the 3 collagen-encoding genes were significantly upregulated at both days 14 and 28. Eln was significantly upregulated after 14 days, and Fbn 1 expression remained unaffected.

As in the PPE model, both pri-miRs (pri-miR-29b-1/29a and primiR-29b-2/29c) were significantly downregulated at both time points $(-1.57$ and -1.64 fold after 14 days, respectively; -1.62 and -1.54 fold after 28 days, respectively; $P<0.05)$ in AngII mice compared with those in sham-operated mice.

miR-29b regulates fibrosis in cultured buman aortic cell lines. Given the importance of various vascular cell types in AAA development, and to confirm that miR-29b is also expressed in human aortic cells, we performed in vitro experiments using non-growth arrested human aortic smooth muscle cells (hASMCs) and human aortic adventitial fibroblasts (hAFBs). Cells were treated with TGF- $\beta 1$, a known regulator of miR-29b, and profibrotic stimulus in different cell lines, such as cardiac fibroblasts (8), tubular kidney cells (7), and hepatocytes (6).

Treatment with TGF- $\beta 1$ significantly decreased miR-29b expression in hAFBs but not in hASMCs (Figure 3A). miR-29b expression was then modified by transfecting with either an antagomir (anti$29 b$ ) to inhibit activity or a pre-miR (pre-29b) to enhance activity in both cell lines. Successful transfection (>50\% of all cells) was confirmed by visual fluorescent microscopic analysis and FACS for labeled tag (data not shown).

Modulation of miR-29b had a significant impact on collagen gene expression (COL1A1 and COL3A1) in both cell types, although the effect in hAFBs was more dramatic (Figure 3, B and C). Overexpression of miR-29b also inhibited expression of $E L N$ in hASMCs (Figure 3C).
Since TGF- $\beta 1$ preferentially altered miR-29b expression in hAFBs, we monitored collagen production after stimulation in order to confirm the profibrotic effect of miR-29b downregulation in this specific cell line. Collagen synthesis was increased in TGF- $\beta 1$-treated cells compared with that in an untreated control group. Anti-29b exacerbated the effects of TGF- $\beta 1$ treatment upon collagen synthesis. In contrast, pre-29b decreased collagen levels (Figure 3D).

In vivo modulation of miR-29b alters collagen gene expression, aneurysm progression, and aortic wall structure in the PPE model. We used FITC-labeled locked nucleic acid (LNA) anti-miR-29b to inhibit miR-29b (anti-29b) or GFP-labeled lentivirus for transduction with pre-miR-29b (lenti-pre-miR-29b; pre-29b) to study gain of function. Double immunofluorescence studies confirmed that anti-29b and pre-29b each successfully incorporated into the aortic wall (Figure 4A). Both miR-29b modulators coexpressed visually with the SMC marker, smooth muscle $\alpha$-actin (SMA), as well as in the adventitial layer. However, localization of miR-29b modulators was almost exclusively limited to the site of injury (aneurysmal part of the abdominal aorta) in the PPE model. In noninjured suprarenal aortic segments from miR-29bmodulated mice, any green color appeared to be largely due to autofluorescence from elastic fibers or adventitial in nature, possibly delivered through vasa vasorum from the injured region. Successful inhibition and overexpression in vivo were furthermore confirmed by quantitative RT-PCR (qRT-PCR) measuring miR-29b expression in anti-29b- and pre-29b-transduced mice, in relation to that of a scrambled control miR (scr-miR; Supplemental Figure 1A). 
A
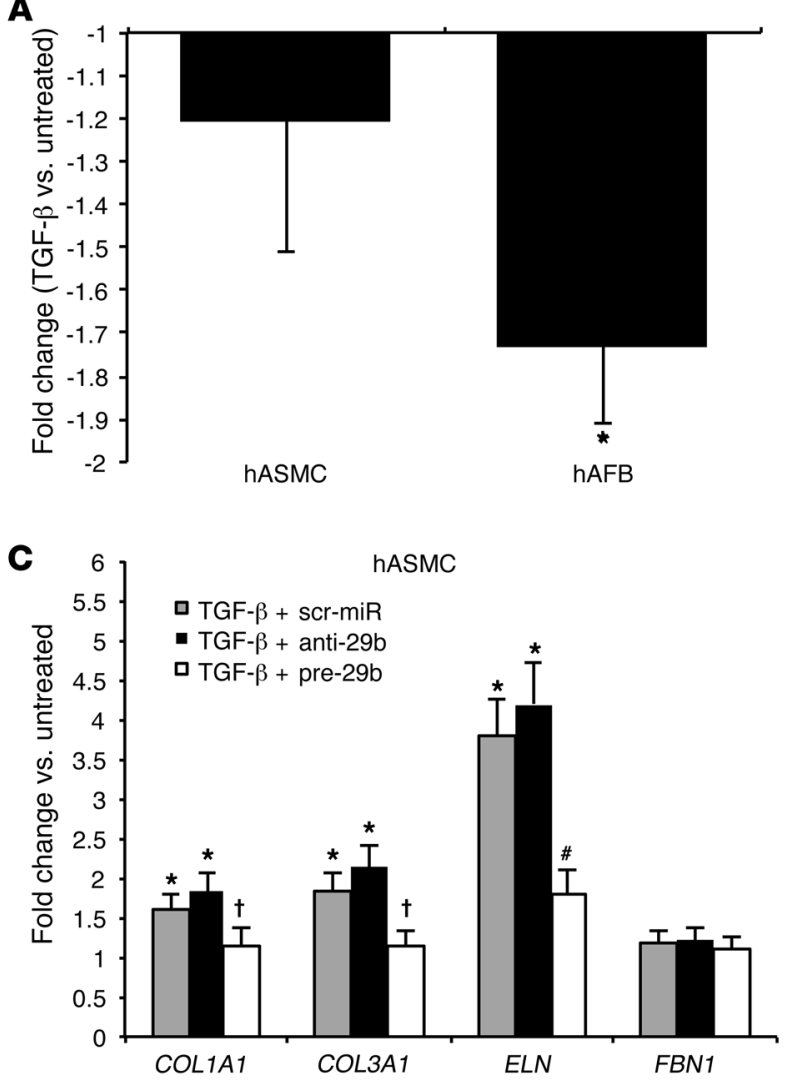

B

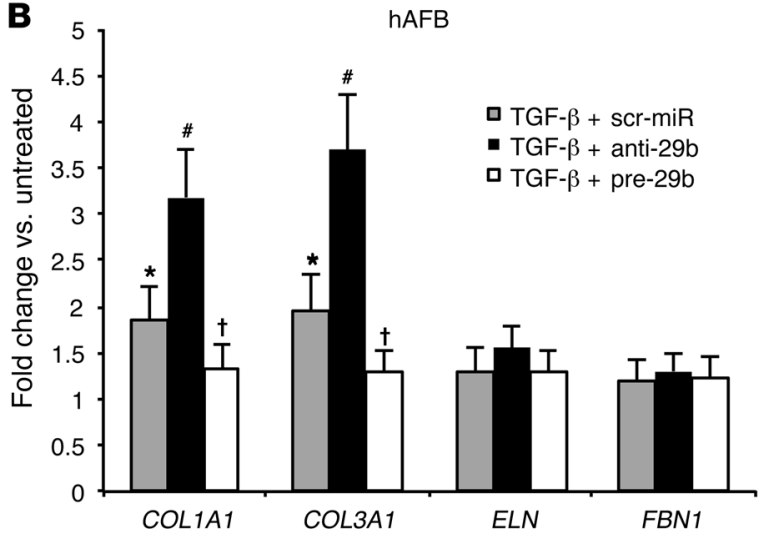

D

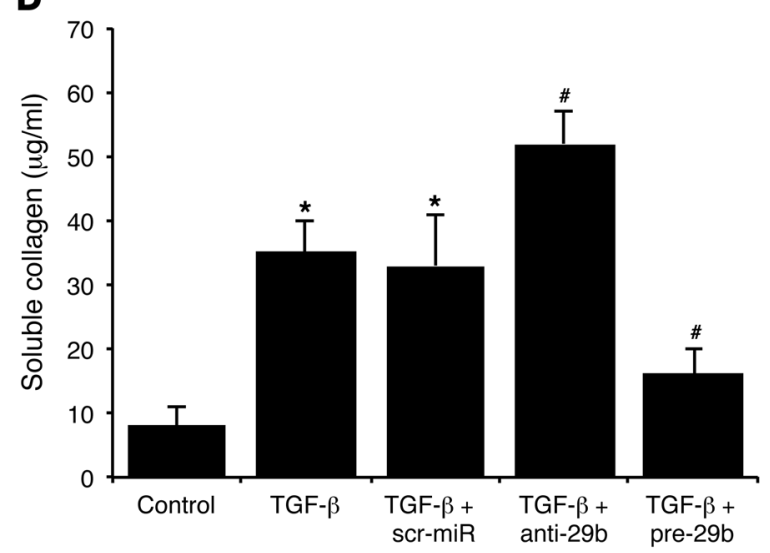

Figure 3

miR-29b in vitro. (A) miR-29b expression in TGF- $\beta$-stimulated hASMCs and hAFBs. (B) Expression levels of miR-29b target genes in TGF- $\beta$-stimulated anti-29b- and pre-29b-transfected hAFBs. (C) Expression levels of miR-29b target genes in TGF- $\beta$-stimulated anti$29 \mathrm{~b}$ - and pre-29b-transfected hASMCs. (D) Soluble collagen assay to quantify collagen synthesis in TGF- $\beta$-stimulated anti-29b- and pre-29b-transfected hAFBs. Data are mean \pm SEM. ${ }^{*} P<0.05$ versus untreated. ${ }^{\#} P<0.05$ versus scr-miR and untreated. ${ }^{\dagger} P<0.05$ versus scr-miR and anti-29b.

Enhancing expression of miR-29b with pre-29b after elastase treatment greatly augmented AAD growth, whereas inhibition with anti-29b inhibited AAD expansion (significant from day 7 to day 28; Figure 4B, Supplemental Figure 1B, and Supplemental Table 3). Interestingly, 4 mice died after receiving pre-29b (2 at day 9 and 1 each at 13 and 21 days), due to rupture of their greatly enlarged AAAs, an extremely uncommon event in the PPE-induced aneurysm model when using 10-week-old male C57BL/6 mice.

Anti-29 (10 mg/kg) significantly increased collagen (Col1a1, Col3a1) and Eln mRNA levels at days 7, 14, and 28 after elastase infusion compared with scr-miR (Figure 4, C-E). In contrast, treatment with lenti-pre-miR-29b (7.6 × $10^{7}$ infection units/ml [IFU/ $\mathrm{ml}]$ ) in ELAST mice led to collagen and elastin gene normalization (nonsignificant compared with that of sham-operated mice) at all 3 time points after tail vein injection. Even scr-miR-injected mice had significantly higher expression of collagen genes and Eln compared with that of pre-29b mice (Figure 4, C-E).

Analogous to our double immunofluorescence results, anti-29b or pre-29b had minimal impact on target gene expression in the suprarenal (non-aneurysmal) abdominal aorta, suggesting that only the site of injury had significant uptake of the miR modulators (Figure 4F).

Polarized light microscopy of picrosirius red-stained aortic cross sections demonstrated substantial effects of anti-29b and pre-29b (compared with those of scr-miR) on PPE-induced aneurysm structure and composition at 28 days. Some enhancement of collagen deposition, indicating vascular fibrosis, was visible in scr-miR mice compared with that in sham-operated mice. This process became much more prominent in anti-29b-injected mice compared with that in the other 3 groups, whereas almost no fibrotic or proliferative response could be detected in the greatly dilated pre-29btreated aortas (Figure 5A). Further, immunohistochemical analysis for SMA (SMC marker), Ki-67 (nonspecific marker of cell proliferation), and Mac-1 (monocyte/macrophage marker) revealed no major differences between miR-29b-modulated mice when compared with scr-miR (Supplemental Figure 1, C-E), with regards to smooth muscle cell proliferation or inflammatory cell infiltration. Cell proliferation (Ki-67-positive cells) appeared to be lower in the adventitial region in pre-29b-transduced mice, in conjunction with the limited fibrotic response, when compared with that in scr-miRand anti-29b-transduced mice.

To confirm the gene expression results for Col3a1 (the most significantly regulated collagen gene), we performed immunohistochemical analysis in scr-miR- as well as anti-29band pre-29b-transfected mice 14 days after AAA induction (Figure $5 B)$. As seen in Figure 5C, Col3a1 was significantly increased in the anti-29b aortic wall compared with that in the other 2 groups. 
A

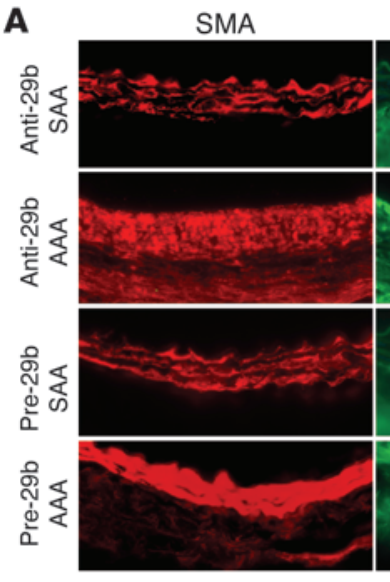

C

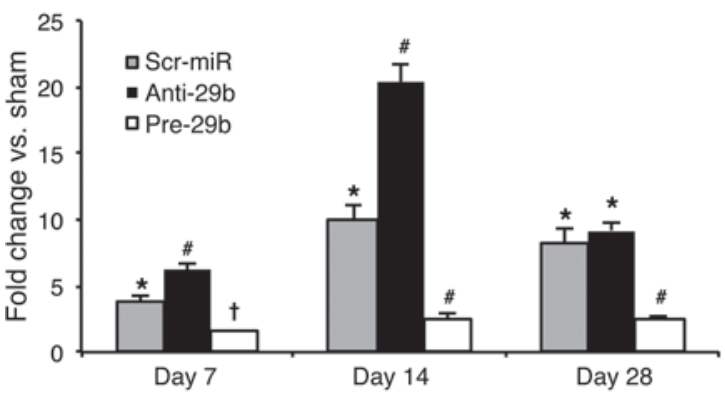

E

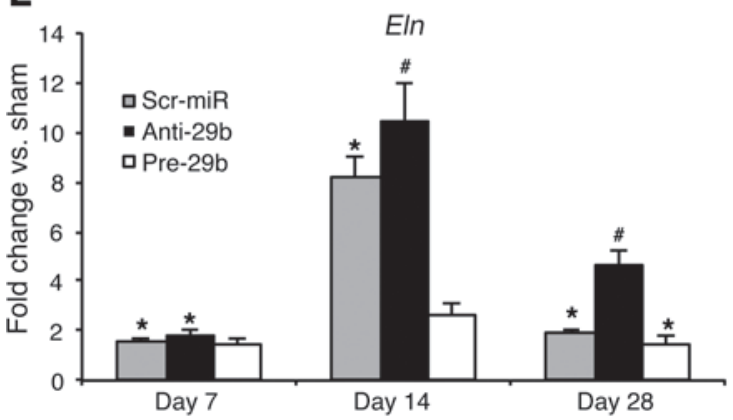

GFP/FITC

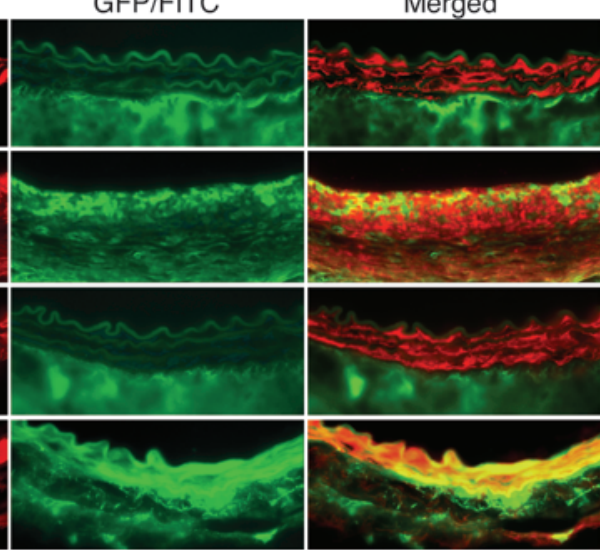

B

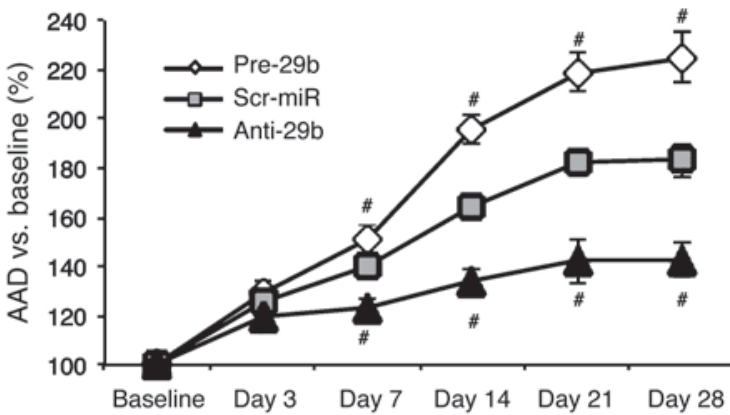

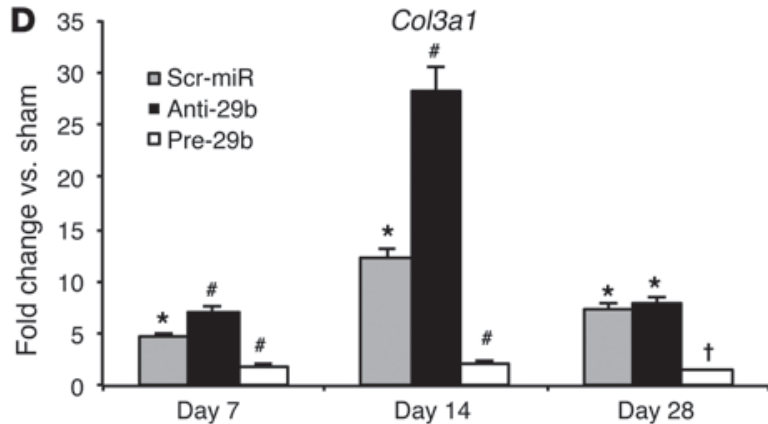

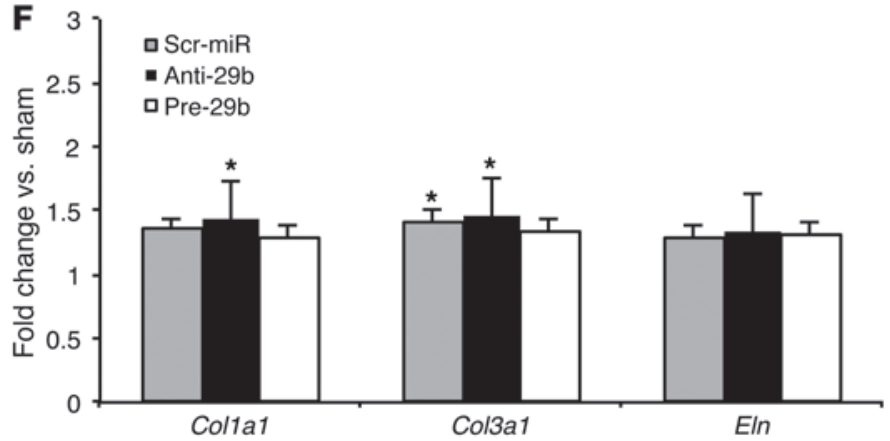

Figure 4

Effects of anti-29b and pre-29b in PPE-AAA. (A) Double immunofluorescence detection of smooth muscle cell $\alpha$-actin (SMA, red) and GFP/ fluorescein isothiocyanate label (green) in the non-aneurysmal part of the suprarenal abdominal aorta and in the infrarenal site of injury (AAA) in anti-21- or pre-21-transduced mice with AAA (as single color and merged images; original magnification, $\times 200$ ). (B) AAD (in percentage versus baseline) in scr-miR and anti-/pre-21 PPE-induced AAA. (C) Col1a1, (D) Col3a1, and (E) Eln expression in scr-miR- and anti- and pre29b-transduced mice compared with that in sham-operated mice in the PPE model. (F) Col1a1, Col3a1, and Eln expression is not significantly different between scr-miR- and anti- and pre-29b-transduced mice in the non-aneurysmal suprarenal aorta in the PPE-AAA model 14 days after induction. $n=4-8$ mice for each time point and group. Data are mean \pm SEM. ${ }^{*} P<0.05$ versus sham. ${ }^{*} P<0.05$ versus scr-miR and sham. $+P<0.05$ versus scr-miR and anti-29b.

MMP activity is regulated through miR-29b expression. Using in situ zymography, we identified varying levels of MMP activity in anti$29 \mathrm{~b}$ - and pre-29b-treated mice compared with those in scr-miRtreated mice. MMP activity appeared lower in anti-29b-treated mice compared with that in the other 2 groups at 14 days after AAA induction using PPE (Figure 5D). In contrast, MMP activity was increased in pre-29b-treated mice with large AADs. qRT-PCR confirmed that Mmp2 and Mmp9 expression was increased in mice with upregulated miR-29b (pre-29b) as compared with that in the other 2 groups (Figure 5, E and F). Mmp2 and Mmp9 expression was significantly decreased in anti-29b-treated mice at the same time point. A similar pattern of MMP2 and MMP9 regulation was observed in TGF- $\beta 1$-stimulated and miR-29b-modulated hAFBs (Supplemental Figure 2, A and B).

In vivo modulation of miR-29b substantially affects $A A A$ expansion and ruptures in the AngII model. In an effort to illustrate similar effects of miR-29b modulation in the second mouse model of AAA induction, the AngII infusion model, we modulated mice with either 

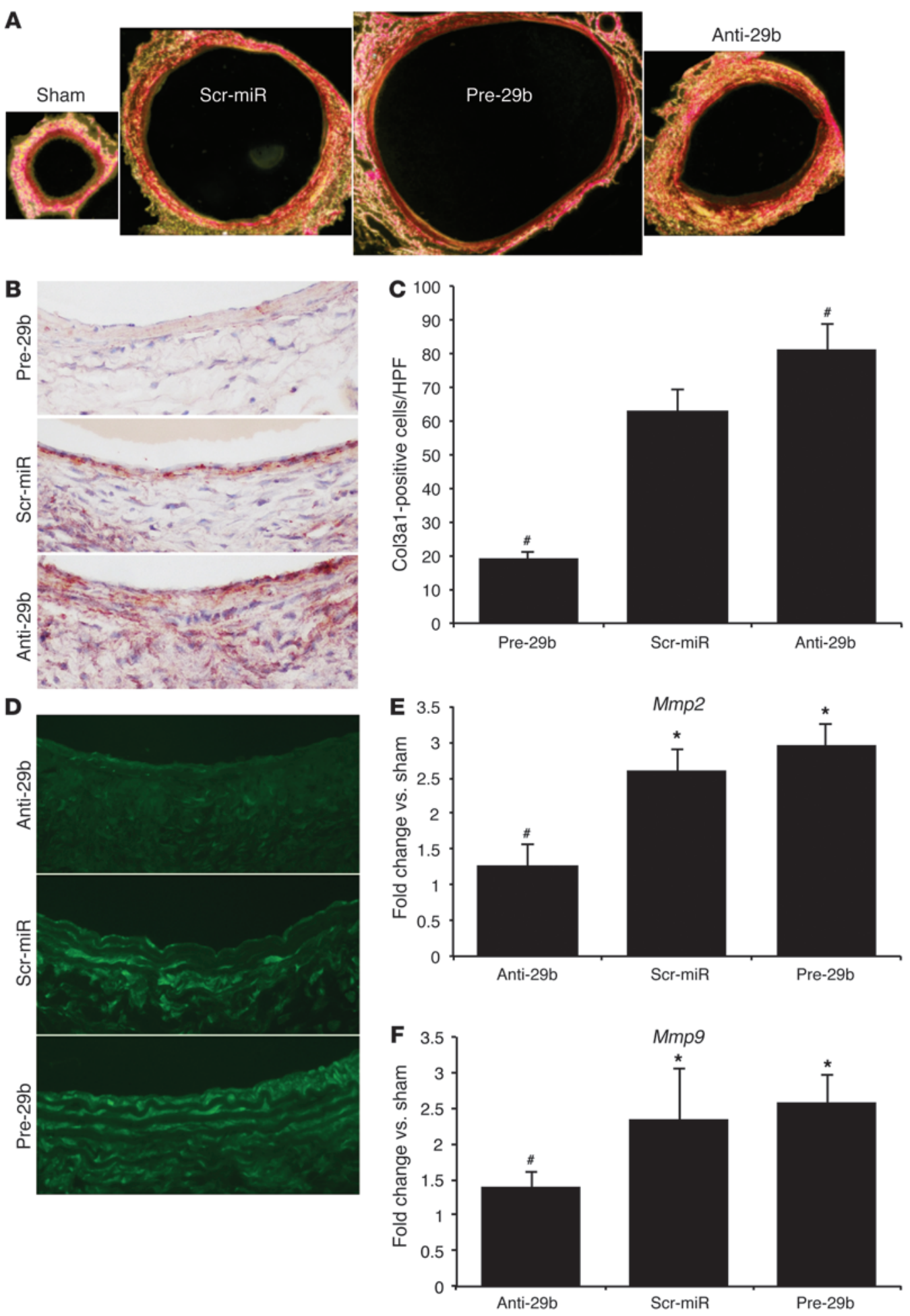

\section{Figure 5}

Fibrosis in miR-29b-modulated mice with PPE-induced AAAs. (A) Representative polarized light microscopy of picrosirius red-stained aortic cross sections (original magnification, $\times 40$ ), illustrating aneurysm expansion and fibrosis (collagen fibers are bright yellow) in sham, scr-miR, and anti- and pre-29b mice 28 days after AAA induction with PPE. (B) Representative Col3a1 immunohistochemical images, demonstrating effects of pre-29b, scr-miR, and anti-29b 14 days after AAA induction with PPE (original magnification, $\times 200)$. (C) Quantification of Col3a1-positive cells in the intima/media region ( $n=4$ high-power fields of 3 different aortas per group) 14 days after AAA induction with PPE. (D) In situ zymography images to detect MMP activity in scr-miR- and anti- and pre-29b-transduced aortas 14 days after PPEinduced AAA in mice (original magnification, $\times 200)$. (E) Mmp2 and (F) Mmp9 expression in miR-29b-modulated mice with PPE-induced AAA (after 14 days). $n=4-8$ mice per treatment group. Data are mean \pm SEM. ${ }^{*} P<0.05$ versus sham. ${ }^{P} P<0.05$ versus scr-miR and anti-29b or pre-29b.

$29 \mathrm{~b}$ significantly decreased target gene expression. No significant change of Col1a1, Col3a1, and Eln regulation was detectable 7 days after AAA induction (Figure 6, B-D). Ruptures occurred significantly more often in pre-29b-treated mice (63\%) compared with those in scrmiR- $(33 \% ; P<0.01)$ and anti-29b-transduced mice $(20 \% ; P<0.01)$ until day 28.

anti- or pre-29b (and compared them to those modulated with scr$\mathrm{miR}$ ) as described above for the PPE model. Further downregulation of miR-29b with anti-29b resulted in a significant decrease of AAA expansion after 28 days. Overexpression of miR-29b by pre-29b significantly increased the AAD 14 and 28 days after AAA induction with AngII (Figure 6A). Expression of Col1a1, Col3a1, and Eln was significantly altered after 14 days. After 28 days, only pre-
However, the overall effect of miR-29b modulation appeared to be more moderate compared with that in the PPE model. Interestingly, miR-29b expression in anti- and pre-29b-modulated mice was not altered as extensively as that in mice in the PPE study (Supplemental Figure 1A and Supplemental Figure 2C). A potential explanation for this may be due to the fact that the acute and localized injury induced in the PPE model likely leads to an increased 


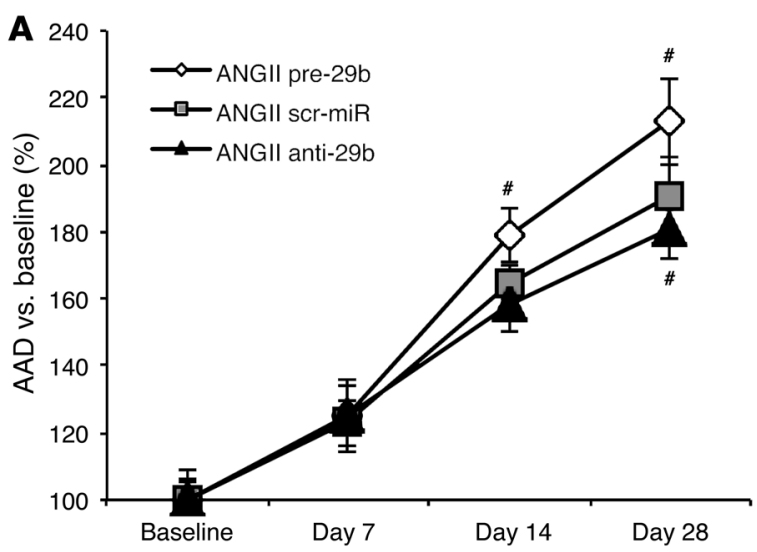

B
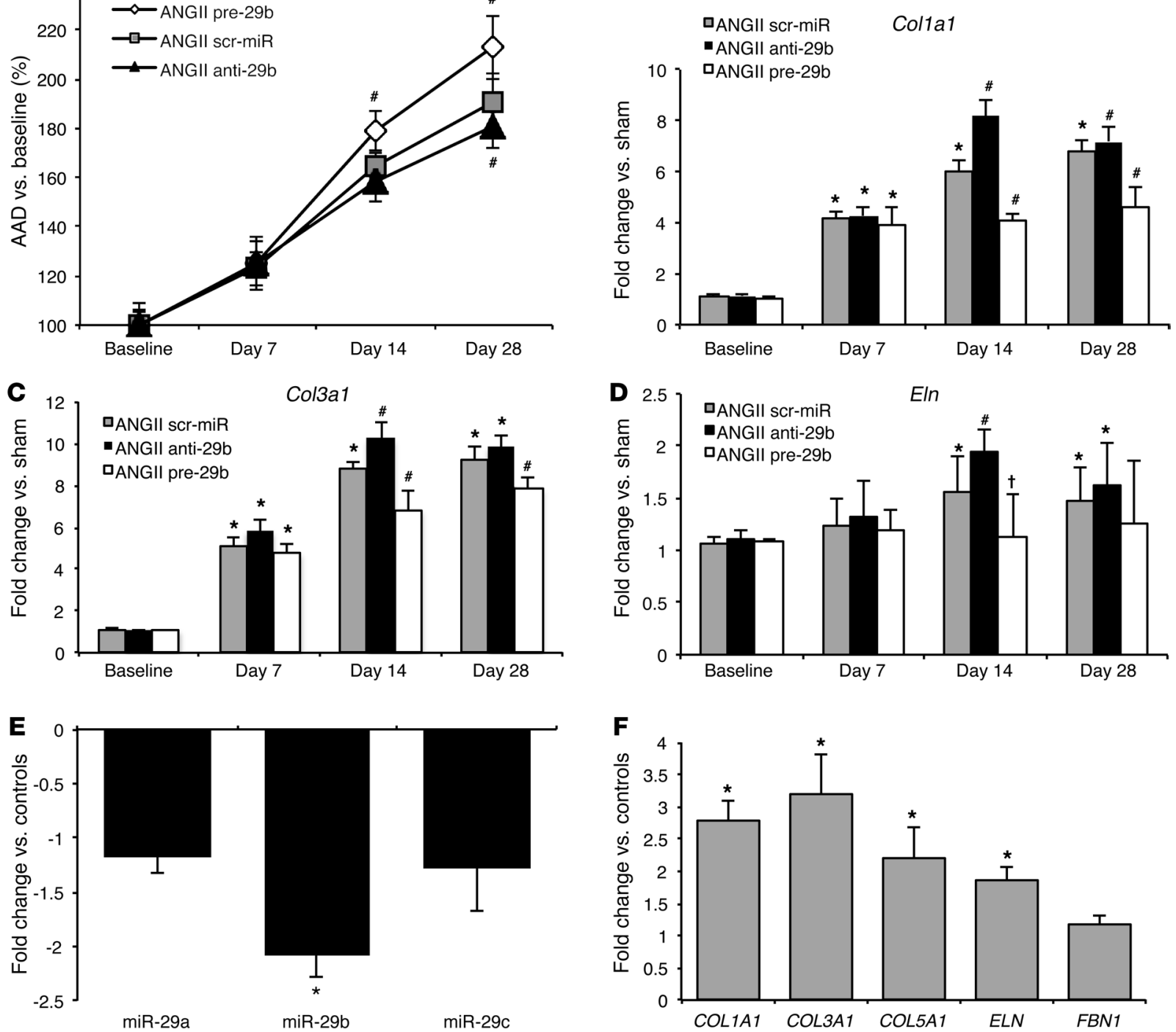

\section{Figure 6}

Effects of anti-29b and pre-29b in Angll infusion model and miR-29b regulation in human patients with AAA. (A) AAD (in percentage versus baseline) in scr-miR and anti- and pre-29b Angll-induced. (B) Col1a1, (C) Col3a1, and (D) Eln expression in scr-miR- and anti- and pre-29b-transduced mice compared with that in sham-operated mice in the Angll model ( $n=4-6$ mice for each time point and group). (E) miR-29b is the only significantly downregulated miR in human AAA samples $(n=8)$ compared with tissue from control patients $(n=7)$ without AAA. (F) COL1A1, COL3A1, COL5A1, and ELN are significantly upregulated in human AAA tissue compared with non-aneurysmal aortic tissue. Data are mean \pm SEM. ${ }^{*} P<0.05$ versus sham or control patients. ${ }^{\#} P<0.05$ versus scr-miR and sham. ${ }^{\dagger} P<0.05$ versus scr-miR and anti-29.

uptake of miR modulators, whereas the intact aortic wall in the AngII model might result in limited uptake of anti- and pre-29b.

Human patients with AAA display downregulated aneurysm tissue miR$29 b$ and upregulated collagen $m R N A$ levels. Human infrarenal aortic tissue samples from patients $(n=15)$ who underwent surgery for replacement of an enlarged abdominal aorta (AAD, 59-68 mm) corroborated our findings of downregulated miR-29b and increased collagen gene expression with aneurysm. We compared this group of patients with AAA (aged $64 \pm 11$ years) with a group of control organ donor patients without AAA $(n=5$, mean age $33 \pm 14$ years) at time of explantation ( $n=3$ heart donors; $n=2$ kidney donors). According to hospital documentation, all 15 patients with AAA were on similar medical therapy, which might have potentially influenced their aortic molecular milieu (beta blockers, either angiotensin receptor blocker or angiotensin-converting enzyme inhibitors, and statin therapy) at the time of surgical intervention. All patients were male, of mixed European descent, and nondiabetic. Similar to that in our preclinical animal models of AAA, qRTPCR revealed that miR-29b was $-2.3 \pm 0.6$ fold downregulated in diseased aortic tissue from patients with AAA. Further, miR-29b was the only member of the miR-29 family found to be significantly regulated in patients with AAA (Figure 6E). COL1A1, COL3A1, COL5A1, and ELN were also all significantly upregulated in AAA (Figure 6F), supporting the data from our murine AAA studies. 


\section{Discussion}

The development of an aneurysm and its progression and ultimate rupture involve complex pathological mechanisms. Identification of underlying causes and successful medical treatment remains a major challenge in modern vascular medicine. New approaches in understanding and fighting AAA disease are considered to be both necessary and inevitable. The identification of what we believe to be an entirely new method of gene regulation through miRs, and validation of miRs as markers and modulators of vascular remodeling in pathological conditions, will potentially provide us with innovative molecular therapies to fight the burden of vascular diseases.

Previously published studies have already indicated that the miR-29 family possesses therapeutic potential in fibrosis-related diseases. A profibrotic response is often considered to be pathologic, accompanied by a significant impact on and malfunction of the affected organ system. miR-29b has been identified to be downregulated and to control cardiac fibrosis after myocardial infarction and in ischemia (8) as well as in liver (6) and renal fibrosis (7). In these conditions, increased collagen gene expression was directly regulated by a decrease in miR-29b levels.

A recent miR profiling study in patients with thoracic aortic dissections indicated that miRs may contribute to thoracic aortic dissection pathogenesis. Conducting miR gene network analysis, the authors concluded that the miR-29 and miR-30 families are very likely to play a key role in regulating 2 aortic dissection-linked pathways, the MAPK and the focal adhesion signaling pathways (16).

Data from our study suggest that downregulation of miR-29b triggers a profibrotic response in AAAs. In this case, strategies to further decrease miR-29b expression might potentially have beneficial effects in protecting the aorta from expansion and ultimate rupture. Antagomirs targeting miR-29b represent an effective means of further decreasing miR-29b expression and thereby inducing an augmented profibrotic response.

The precise mechanisms that lead to repression of miR-29b expression in experimental AAA development remain to be further investigated, but our results support other previously published work (6-8), indicating that TGF- $\beta$, a validated regulator of tissue fibrosis, can repress miR-29b expression. The role of TGF- $\beta$ signaling in aneurysm development remains controversial. A nuanced assessment suggests opposing roles of canonical (Smad-based) signaling and noncanonical (ERK/JNK-based) signaling. Overactivity of TGF- $\beta$ has been postulated to be a cause of thoracic aortic aneurysms associated with Marfan syndrome (17). However, recent work points to noncanonical TGF- $\beta$ signaling as being the probable primary driver of aneurysm in these mice, with canonical signaling playing a lesser but protective role (18). The protective aspects of TGF- $\beta$ canonical signaling are supported by the identification of a frameshift mutation in the SMAD3 gene in humans that induces familial thoracic aortic aneurysm formation and dissection as well as intracranial and other arterial aneurysms (19). Another study identified TGF- $\beta$ as a critical regulator of the innate immune response and preserver of vessel wall integrity in AngII-induced murine AAAs (20). Further, Apoe-/- mice that overexpress TGF- $\beta$ have reduced aortic root aneurysm development compared with that of nontransgenic littermate controls (21). Lastly, TGF- $\beta$ overexpression has been shown to limit established AAA progression and inflammatory processes (22). Our study supports the protective role of TGF- $\beta$ in limiting AAA disease and offers a miR-29-based mechanism.
Because of its general role in fibrosis, there have been numerous efforts to therapeutically target TGF- $\beta$. However, this has been problematic because of the numerous additional functions ascribed to TGF- $\beta$, for example in modulating immune response (23). Data from our in vitro experiments show that the profibrotic effects of miR-29b downregulation and TGF- $\beta$ stimulation in aortic cells are mediated primarily by fibroblasts. However, modulation of miR$29 \mathrm{~b}$ levels in smooth muscle cells, especially overexpression with pre-29b, resulted in significant alteration of target gene expression as well. Our in vivo data suggest that both miR-29b-1 and miR-29b2 contribute to the observed levels of downregulation in AAA.

Collagen gene expression, and to lesser extent elastin, were most consistently affected by miR-29b modulation. FBN1, considered an important component of the ECM, remained almost unaffected by transduction in vivo and transfection in vitro. In addition, the effect of systemic administration of anti- and pre-29b on collagen mRNA levels in the aorta was relatively minor in comparison to much greater effects in heart, liver, and kidney (Supplemental Figure 2, D-F). Agents that modulate miR-29b expression appear to be relatively more efficient in these organs, likely due to increased uptake in comparison with that in the aorta. Fibrosis in these other organs is unfortunately associated with serious disease. These findings suggest that systemic downregulation of miR-29b will not be useful per se for translational approaches in humans. However, local delivery with expandable balloons and/or drugeluting stents containing anti-miR-29b may emerge as a promising avenue to trigger fibrosis in the aortic wall in human patients with early AAA disease. Experimental delivery of siRNA molecules eluted from stents and balloons has already been achieved (24).

Our studies suggest that downregulation of miR-29b is a physiologic response of the aortic wall to expansion. Increased collagen gene expression and augmented fibrosis substantially inhibited AAA expansion, indicating that miR-29b represents a potential therapeutic target. While prediction algorithms and recently published studies on liver and prostate cancer $(19,20)$ suggest that MMP2 and MMP9 are targets of miR-29b, it is interesting to note that we observed an inhibition of gelatinase activity with anti-miR-29b treatment. This speaks to the complex regulation of metalloproteinase activity in general as well as a potential regulation through miR-29b in AAA disease specifically.

The fact that inhibition of a single miR (i.e., miR-29b) can induce significant perivascular fibrosis of the aortic wall and thereby protect the aorta from expansion shows the power of individual miRs in regulating numerous target genes in a coordinated fashion (Supplemental Figure 3). Unlike most traditional therapeutic approaches in which drugs have specific cellular targets, the key component of miR modulation lies in regulation of entire functional gene networks (25). However, this is also a potential weakness of the approach, as unintended off-target effects may occur.

In chronic diseases such as AAA, long-term administration and repeated agent delivery might be necessary to provide at-risk patients with an effective treatment. LNA-anti-miR administration could have limited utility, if the route of delivery requires an invasive procedure. In our study, changes in collagen gene expression by the LNA-anti-miR-29b could not be detected at 28 days. Clearly, examination of extended time frames of miR modulation is needed.

Vascular gene therapy was the other major approach used in our experiments. Lentivirus, derived from HIV-1, appears to be a useful tool to deliver miRs to the injured and expanding aorta. Production of wild-type HIV through homologous recombination of len- 
tiviral vectors is a major safety concern (26). However, the lentiviruses used in our study resolve this issue. The U3 promoter region of the long terminal repeats is deleted, leading to self-inactivation of the virus (27). The small size of miR coding sequences within a single dose should simplify the delivery of either pre-miRs or antagomirs. This makes them a promising vector for future applications beyond vascular cell therapy (28). While further investigations into miR biology and functionality are clearly required, the future of miRs and their therapeutic potential in limiting and inhibiting cardiovascular diseases is closely bound to the optimization of gene delivery vectors.

\section{Methods}

PPE infusion model. The PPE infusion model to induce AAA was performed as previously described (29). In brief, after placing temporary ligatures around the proximal and distal aorta, an aortotomy was created at the bifurcation, and an insertion catheter was used to infuse the aorta for 5 minutes at $100 \mathrm{mmHg}$ with saline or saline containing type I PPE $(1.5 \mathrm{U} / \mathrm{ml}$; Sigma-Aldrich). After removing the infusion catheter, the aortotomy was repaired without constriction of the lumen. At 7, 14, or 28 days, 2 aortic segments were harvested: the induced AAA (area between the left renal artery and the bifurcation) and the suprarenal abdominal aorta (area proximal to the renal arteries up to the diaphragm). Samples were snap frozen in liquid nitrogen and then stored at $-80^{\circ} \mathrm{C}$ pending further processing. A total of 125 mice was used for AAA induction using PPE (or sham). The survival rate up to day 28 (if applicable) was more than $80 \%$. The mortality rate was slightly increased in pre-29b-transduced animals compared with that in all other groups (see Results).

AngII infusion model. Osmotic pumps (model 2004, Alzet) containing either AngII $(1 \mu \mathrm{g} / \mathrm{kg} / \mathrm{min}$, Sigma-Aldrich; $n=34)$ or saline $(n=16)$ were introduced in 10-week-old Apoe - $^{-}$male mice (C57BL/6J background) as previously described (30). Aneurysmal segments of the aortas (proximal to the renal arteries) were harvested after 14 or 28 days, snap frozen in liquid nitrogen, and then stored at $-80^{\circ} \mathrm{C}$ pending further processing.

Aortic diameter measurements by ultrasound imaging. At baseline and 3, 7, 14,21 , and 28 days after aneurysm induction, B-mode ultrasound imaging was performed on the operated mice to assess the $\mathrm{AAD}$ as previously described (31). Measurements were accomplished using random selection of each data set, and the operators were blinded to prevent recall bias. All measurements were collected by 1 observer to limit operator variation, while results were analyzed by a second independent observer blinded to the treatment group.

Histological and immunohistochemical analysis. Histological staining after harvesting was performed in the same region of abdominal aorta that was imaged in order to obtain morphometric data to correlate with ultrasound measurements and gene expression results from qRT-PCR using a standardized protocol as previously described (31). Aortic tissue was divided into 4 (spaced 0.5 -mm apart) 7- $\mu \mathrm{m}$ thick serial sections from the left renal artery to the bifurcation and stained with picrosirius red (Sigma-Aldrich) and with rabbit antibodies against Col3a1, SMA, Ki-67, and Mac-1 (all from Abcam) using the Vectastain ABC Kit (Vector Laboratories) for immunohistochemical analysis. Col3a1-positive cells were measured by counting the cells in 4 high-power fields of 3 different aortas per treatment group. Counting was performed by a blinded investigator 14 days after PPE infusion to induce AAA. All histological analyses were obtained at room temperature using a Zeiss Axioplan 2 (Carl Zeiss MicroImaging) with Zeiss Achroplan and Zeiss Plan-Neofluar lenses, a Nikon Digital Sight (DS) Ri1 camera, and the NIS-Elements F 3.00 software (Nikon). Rotating filters (Nikon) were used for polarized light microscopy in picrosirius red-stained images to detect the presence of collagen fibers.
RNA quantification. Total RNA was isolated using a TRIzOL-based (Invitrogen) RNA isolation protocol. RNA was quantified by Nanodrop (Agilent Technologies), and RNA and miRNA quality were verified using an Agilent 2100 Bioanalyzer (Agilent Technologies). Samples required 260/280 ratios of more than 1.8 , and sample RNA integrity numbers of more than 9 for inclusion. RNA was reverse transcribed using the TaqMan microRNA Reverse Transcription Kit (Applied Biosystems) according to the manufacturer's instructions. MicroRNA and TaqMan Assay Kits (Applied Biosystems) for hsa-miR-29a, hsa-miR29b, hsa-miR-29c, pri-miR-29b-1/29a, pri-miR-29b-2/29c, and sno202 (endogenous control for normalization in mice) and RNU44 (for human samples) were used. For mRNA, the iScript cDNA Synthesis Kit (Bio-Rad) was used to synthesize first-strand cDNA according to the manufacturer's protocol. TaqMan qRT-PCR assay was performed using mouse and human specific primers (Applied Biosystems) for COL1A1, COL3A1, COL5A1, ELN, FBN1, MMP2, and MMP9. All probes were normalized to $18 \mathrm{~S}$ as internal control. Amplification took place on an ABI PRISM 7900HT (Applied Biosystems). All fold changes were calculated by the method of $\Delta \Delta \mathrm{Ct}$ and are expressed as mean \pm SEM compared with sham-operated mice, saline-infused mice, saline-treated cells (in vitro), or human control patients (as indicated). All experiments included 5-12 samples per group and time point.

ISH. ISH for miR-29b was performed by using the miRCURY LNA microRNA ISH Optimization Kit (Exiqon) and $5^{\prime}$-DIG- and $3^{\prime}$-DIGlabeled probes for mmu-miR-29b according to the manufacturer's protocol. The sequence of the LNA miR-29b control probe was 5'-DIG/AACACTGATTTCAAATGGTGCTA/DIG-3'.

LNA-anti-miR-29b injection. Either an LNA-anti-miR-29b or scr-miR (miRCURY LNA miR inhibitor from Exiqon) was injected via tail vein under pressure (in $1 \mathrm{ml}$ of PBS over 5-10 seconds). The concentration of anti-miR or scrambled miR was $10 \mathrm{mg} / \mathrm{kg}$. A single injection of anti-miR was performed 1 day after AAA induction both murine AAA models. The custommade LNA-anti-miR-29b 5'-3' sequence was ACTGATTTCAAATGGTGCT. The LNA scrambled miR control 5' $-3^{\prime}$ sequence was GTGTAACACGTCTATACGCCCA.

Lenti-pre-miR-29b injection. The miR-29b pre-miR construct (System Biosciences) was cloned into a HIV (12) lentiviral vector containing a copGFP reporter with the miR precursor under constitutive CMV promoter control. The precursor miR-29b sequence was GGTACCGTTGTCTTGGGTTTATTG. Lenti-pre-miR-29b was injected via tail vein in $1 \mathrm{ml}$ PBS with $7.6 \times 10^{7} \mathrm{IFU} /$ mouse of loaded lentivirus. Lenti-pre-miRs were injected 1 day after AAA induction.

Double immunofluorescence studies. Primary antibody for GFP was applied after washing with $10 \%$ PBS using conventional IHC dilutions (see Supplemental Methods). Secondary antibodies were replaced with goat anti-rabbit antibodies labeled with Alexa Fluor (Invitrogen; dilution 1:250) dye, with a maximum excitation at $488 \mathrm{~nm}$ (green). Polyclonal rabbit SMA primary antibody was detected using a goat anti-mouse secondary antibody labeled red with Alexa Flour 568 (Invitrogen). Images were obtained and analyzed by fluorescence microscopy (microscope and camera from Nikon).

In situ zymography. In situ zymography was performed according to manufacturer's instructions and as described elsewhere (32). In brief, aortic sections from scr-miR- as well as anti-29b- and pre-29b-transduced mice were exposed to DQ gelatin $(10 \mu \mathrm{g} / \mathrm{ml}$; Invitrogen) and $1 \%$ agarose solution (ratio 1:10). Representative images were obtained after digestion of highly fluorescein-labeled gelatin by the endogenous gelatinases MMP2 and MMP9.

In vitro studies. hASMCs and hAFBs were propagated in growth media (SmGM-2 [for hASMCs] and SCBM [for hAFBs]) with 5\% fetal bovine serum as per manufacturer's instructions (Lonza; passage no. 4-5). Primary human cells were derived from the donor aortic arch region (per the manufacturer). For in vitro experiments, cells were treated with human 
TGF- $\beta 1$ ( $2.5 \mathrm{ng} / \mathrm{ml}$; R\&D Systems) for 24 hours. Cell containing plates were harvested for RNA analysis at approximately $90 \%$ confluence.

Transfection of cultured cells. Transfection of hASMCs and hAFBs was performed using Lipofectamine RNAiMAX (Invitrogen) reagent, mixed with anti-hsa-miR-29b, pre-hsa-miR-29b, or scrambled controls (Ambion). For each transfection, $120 \mathrm{pmol}$ of anti-miR or pre-miR was diluted in $250 \mu \mathrm{l}$ Opti-MEM (Lonza). Two hours after transfection, cells were treated with TGF- $\beta 1$ and harvested 24 hours later. RNA was extracted using TRIzOL (Invitrogen). Successful transfection (>50\% of all cells) was confirmed by visual fluorescent microscopic analysis and FACS sorting for fluorescently labeled control anti-miR and pre-miR.

Soluble collagen assay. Soluble collagen in cultured hAFBs was determined according to the manufacturer's instructions (QuickZyme). In brief, after 24 hours treatment with TGF- $\beta$ and transfection with scr-miR, pre-29b, or anti-29b, conditioned culture medium was collected and centrifuged to remove cell debris. Collagen in solution reacted with Sirius Red color dye and was precipitated in a 96-well plate. Reading was performed on a Versa Max Tunable Microplate Reader (Molecular Devices). The experiment was run in quadruplicate.

Human sample acquisition and preparation. Human samples from patients who underwent surgical repair of their AAA and abdominal aortic samples from organ donors were harvested during surgery (or explantation), snap frozen, and stored at $-80^{\circ} \mathrm{C}$.

Statistics. Data are presented as mean \pm SEM. Groups were compared using 2-tailed Student's $t$ test for parametric data. When comparing multiple groups, data were analyzed by ANOVA with Bonferroni's post-hoc test. Sequential measurements (AADs at consecutive time points) were analyzed by 1-way repeated-measures ANOVA. A $P$ value of equal to or less than 0.05 was considered statistically significant.
Study approval. Approval for studies on human tissue samples was obtained from the Stanford University School of Medicine. Protocols complied with all guidelines and policies of the Stanford University School of Medicine and the Declaration of Helsinki, and all individuals provided informed consent. All animal protocols were approved by the Administrative Panel on Laboratory Animal Care at Stanford University (http://labanimals.stanford.edu/) and followed the NIH and USDA Guidelines for Care and Use of Animals in Research. All experiments were performed with 10-week-old male C57BL/6 (PPE model) mice and 10-week-old male Apoe $/-$ mice on a C57BL/6 background (AngII infusion model). Animals were purchased from The Jackson Laboratory.

\section{Acknowledgments}

We would like to thank A. Connolly, B.A. Dake, N. Kimura, H. Kosuge, and T. Kitagawa for expert technical assistance. This work is supported by research grants from the NIH (1P50HL083800-01 to P.S. Tsao, R.L. Dalman, and M.V. McConnell; 5K08 HL080567 to J.M. Spin), the California Tobacco-Related Disease Research Program (18XT-0174 to P.S. Tsao), the Deutsche Forschungsgemeinschaft (MA4688/1-1 to L. Maegdefessel), the American Heart Association (09POST2260118 to L. Maegdefessel), and the Deutsche Herzstiftung e.V. (S/02/11 to D.R. Merk).

Received for publication October 24, 2011, and accepted in revised form December 14, 2011.

Address correspondence to: Philip S. Tsao, Stanford University School of Medicine, Division of Cardiovascular Medicine, 300 Pasteur Drive, Stanford, California 94305-5406, USA. Phone: 650.498.6317; Fax: 650.725.2178; E-mail: ptsao@stanford.edu.
1. Weintraub NL. Understanding abdominal aortic aneurysm. N Engl J Med. 2009;361(11):1114-1116.

2. Creager MA, et al. Atherosclerotic Vascular Disease Conference: Writing Group V: medical decision making and therapy. Circulation. 2004;109(21):2634-2642.

3. Brady AR, Thompson SG, Fowkes FG, Greenhalgh RM, Powell JT. Abdominal aortic aneurysm expansion: risk factors and time intervals for surveillance. Circulation. 2004;110(1):16-21.

4. Thompson RW, Liao S, Curci JA. Vascular smooth muscle cell apoptosis in abdominal aortic aneurysms. Coron Artery Dis. 1997;8(10):623-631.

5. Zhang C. MicroRNomics: a newly emerging approach for disease biology. Physiol Genomics. 2008;33(2):139-147.

6. Roderburg C, et al. Micro-RNA profiling reveals a role for miR-29 in human and murine liver fibrosis. Hepatology. 2011;53(1):209-218.

7. Qin W, et al. TGF-\{beta\}/Smad3 Signaling Promotes Renal Fibrosis by Inhibiting miR-29. J Am Soc Nephrol. 2011;22(8):1462-1474.

8. van Rooij E, et al. Dysregulation of microRNAs after myocardial infarction reveals a role of miR29 in cardiac fibrosis. Proc Natl Acad Sci U S A. 2008;105(35):13027-13032.

9. De Paepe A, Nuytinck L, Hausser I, Anton-Lamprecht I, Naeyaert JM. Mutations in the COL5A1 gene are causal in the Ehlers-Danlos syndromes I and II. Am J Hum Genet. 1997;60(3):547-554.

10. Rahkonen $\mathrm{O}$, et al. Mice with a deletion in the first intron of the Colla1 gene develop age-dependent aortic dissection and rupture. Circ Res. 2004; 94(1):83-90.

11. Menashi S, Campa JS, Greenhalgh RM, Powell JT. Collagen in abdominal aortic aneurysm: typing, content, and degradation. J Vasc Surg. 1987; 6(6):578-582.

12. Rizzo RJ, et al. Collagen types and matrix protein content in human abdominal aortic aneurysms.
J Vasc Surg. 1989;10(4):365-373.

13. Chen KC, et al. OxLDL upregulates microRNA-29b, leading to epigenetic modifications of MMP-2/ MMP-9 genes: a novel mechanism for cardiovascular diseases. FASEB J. 2011;25(5):1718-1728.

14. Thompson RW, Curci JA, Ennis TL, Mao D, Pagano MB, Pham CT. Pathophysiology of abdominal aortic aneurysms: insights from the elastase-induced model in mice with different genetic backgrounds. Ann N Y Acad Sci. 2006;1085:59-73.

15. Daugherty A, Cassis LA. Mouse models of abdominal aortic aneurysms. Arterioscler Thromb Vasc Biol. 2004;24(3):429-434.

16. Liao $\mathrm{M}$, et al. A microRNA profile comparison between thoracic aortic dissection and normal thoracic aorta indicates the potential role of microRNAs in contributing to thoracic aortic dissection pathogenesis. J Vasc Surg. 2011;53(5):1341-1349.

17. Lindsay ME, Dietz HC. Lessons on the pathogenesis of aneurysm from heritable conditions. Nature. 2011;473(7347):308-316.

18. Holm TM, et al. Noncanonical TGFbeta signaling contributes to aortic aneurysm progression in Marfan syndrome mice. Science. 2011;332(6027):358-361.

19. Regalado ES, et al. Exome sequencing identifies SMAD3 mutations as a cause of familial thoracic aortic aneurysm and dissection with intracranial and other arterial aneurysms. Circ Res. 2011; 109(6):680-686.

20. Wang Y, et al. TGF-beta activity protects against inflammatory aortic aneurysm progression and complications in angiotensin II-infused mice. J Clin Invest. 2010;120(2):422-432.

21. Frutkin AD, et al. TGF-[beta]1 limits plaque growth, stabilizes plaque structure, and prevents aortic dilation in apolipoprotein E-null mice. Arterioscler Thromb Vasc Biol. 2009;29(9):1251-1257.

22. Dai J, et al. Overexpression of transforming growth factor-beta 1 stabilizes already-formed aortic aneu- rysms: a first approach to induction of functional healing by endovascular gene therapy. Circulation. 2005;112(7):1008-1015.

23. Nguyen TL, Sullivan NL, Ebel M, Teague RM, Dipaolo RJ. Antigen-specific TGF-\{beta\}-induced regulatory $T$ cells secrete chemokines, regulate $T$ cell trafficking, and suppress ongoing autoimmunity. J Immunol. 2011;187(4):1745-1753.

24. San Juan A, et al. Development of a functionalized polymer for stent coating in the arterial delivery of small interfering RNA. Biomacromolecules. 2009;10(11):3074-3080.

25. Mishra PK, Tyagi N, Kumar M, Tyagi SC. MicroRNAs as a therapeutic target for cardiovascular diseases. J Cell Mol Med. 2009;13(4):778-789.

26. Cockrell AS, Kafri T. Gene delivery by lentivirus vectors. Mol Biotechnol. 2007;36(3):184-204.

27. Miyoshi H, Blomer U, Takahashi M, Gage FH, Verma IM. Development of a self-inactivating lentivirus vector. J Virol. 1998;72(10):8150-8157.

28. Gray SJ, Samulski RJ. Optimizing gene delivery vectors for the treatment of heart disease. Expert Opin Biol Ther. 2008;8(7):911-922.

29. Azuma J, Asagami T, Dalman R, Tsao PS. Creation of murine experimental abdominal aortic aneurysms with elastase. J Vis Exp. 2009;29(pii): 1280.

30. Goergen CJ, et al. Influences of aortic motion and curvature on vessel expansion in murine experimental aneurysms. Arterioscler Thromb Vasc Biol. 2011;31(2):270-279.

31. Azuma J, Maegdefessel L, Kitagawa T, Dalman RL, McConnell MV, Tsao PS. Assessment of elastase-induced murine abdominal aortic aneurysms: comparison of ultrasound imaging with in situ video microscopy. J Biomed Biotechnol. 2011; 2011:252141

32. Zhang J, et al. Molecular imaging of activated matrix metalloproteinases in vascular remodeling. Circulation. 2008;118(19):1953-1960. 